\title{
Questioning the benefits of current screening protocols for common cancers
}

\author{
Stefan Rodic, Ariel Gershon \\ Faculty Reviewer: George Kim MD, MCISc(FM), CCFP (Department of Family Medicine)
}

\section{ABSTRACT}

Screening is the application of a diagnostic test to a population of patients without suspicious clinical signs or symptoms of a disease. Applying screening protocols to detect cancer is especially attractive as cancer is a growing burden and earlier detection of malignancies can lead to better outcomes. While the initial excitement over screening for cancers has caused protocols to be put into practice, data analysis has cast doubt on the net benefit. Prostate-specific antigen (PSA) blood testing has been used as a screening test for prostate cancer. The harms of a positive PSA screen include invasive investigations and treatments with side effects such as infection and incontinence. The poor sensitivity and specificity of PSA screening caused the Canadian Task Force on Preventive Health Care to recommend against PSA as a screening tool. Similarly, mammography and ultrasound for detecting breast cancer lesions in asymptomatic women suffer from relatively low sensitivity and specificity. The harms of screening include emotional stress and invasive surgical procedures. Meta-analyses reveal that the risks outweigh the benefits for some age groups, but there may be a role in screening asymptomatic patients with a family history of breast cancer with more sensitive but more expensive modalities, such as magnetic resonance imaging (MRI). Finally, colonoscopy, which is the gold standard to diagnose colorectal cancers, has failed to show net benefit in screening. While screening protocols thus far have provided underwhelming results, the key in screening in the future may lie in using tests with high specificity and sensitivity, as in next generation screening modalities that rely on molecular markers and analytics.

\section{INTRODUCTION}

Health Canada estimates that the number of breast, colorectal and prostate cancer cases will increase by $46 \%, 78 \%$ and $96 \%$ respectively, after comparing the number of cancers in 2003 to 2007 with anticipated rates in 2028 to 2032. A large part of these increases can be attributed to an aging Canadian population. ${ }^{1}$

Screening measures have been implemented to reduce cancer mortality based on the assumption that cancers that are detected early can be treated more effectively. However, despite large amounts of funding and widespread acceptance of cancer screening protocols among physicians, the net benefit of these measures is controversial.

Two notable publications examined randomized control trials and meta-analyses that evaluated the mortality benefit of screening for various cancers. They concluded that screening asymptomatic patients offered no overall mortality benefit, despite causing a decrease in disease-specific mortality. ${ }^{2,3}$ It is possible that the examined studies did not have enough power to detect a modest decrease in overall mortality. Alternatively, the screening protocols themselves may have detrimental side effects that counteract their benefits. For example, mass screening can lead to over-diagnosis and an increase in invasive biopsies, surgeries and treatments that have potential side effects. We further examine current screening techniques of asymptomatic patients as well as their efficacy in prostate, breast and colorectal cancers below.

\section{PROSTATE CANCER}

Prostate-specific antigen (PSA) testing is a quantitative measure with variable advantages depending on the cut-off value used for determination of clinically relevant pathology. At a standard cut-off value of $4 \mathrm{ng} / \mathrm{mL}$, the sensitivity of this test is only $20.5 \%$, with $93.6 \%$ specificity. Therefore, it is likely that the vast majority of prostate cancers remain undetected using standard screening techniques. ${ }^{4}$ Nevertheless, it was believed that PSA screening could be effective if administered on a large scale, which is supported by epidemiological evidence. Prostate cancer screening was found to reduce prostate cancer-related deaths by $20 \%$ over a 13 -year period. ${ }^{5}$

However, government-sponsored guidelines have recently been revised to recommend against PSA-based prostate cancer screening. Both the U.S. Preventive Services Task Force and Canadian Task Force on Preventive Health Care have now advised against screening in the general population. ${ }^{6,7}$ The Canadian stance was based on findings that generalized PSA screening did not clearly provide a significant benefit within any age group, but was associated with a proven increase in adverse effects on patients.

The U.S. implemented similar guidelines (against PSA screening), and a study has found that there has been a decrease in absolute number of biopsies, and an increase in the proportion of high risk cancer diagnosed through biopsy. ${ }^{8}$

The relatively benign nature of prostate cancer compared to other malignancies is a key factor in screening considerations. Prevalence of some level of undiagnosed prostate cancer in autopsies increases with age and reaches $70 \%$ in men aged 70 to 79 years. ${ }^{7}$ Many men die from unrelated diseases before prostate cancer has any clinical effect, supporting the notion that some asymptomatic lowgrade cases do not require treatment. Overdiagnosis and aggressive treatment can introduce many risks including bleeding, infection, erectile dysfunction and urinary incontinence. Additionally, many patients are not advised by their healthcare provider on the true efficacy of PSA testing or the variable necessity of treatment. ${ }^{9}$ One 
solution may be to repeat abnormal PSA testing, since it is associated with a decreased risk of biopsy and cancer diagnosis and may be an alternative to eliminating the screening protocol completely. ${ }^{10}$

\section{BREAST CANCER}

Mammography is the primary screening tool used for breast cancer, with a sensitivity of $75 \%$ to $77 \%$ and a specificity of $91 \%$ to 94\% for asymptomatic women. ${ }^{11,12}$ Adding in adjunctive ultrasound screening after mammography can increase the sensitivity from $77 \%$ to $91 \%$, while the specificity is only slightly decreased. ${ }^{12}$

The evidence supporting mammographic screening is controversial, with different studies reaching different conclusions. Invitation to mammography screening may reduce deaths from breast cancer by $25 \%$ to $28 \%$, with one death prevented for every 368 women invited for screening..$^{13}$ On the other hand, retrospective trend analyses across European countries have found indications that mammography has little effect on mortality, and that decreases in mortality are not directly related to screening implementation. ${ }^{14,15}$ Unconventional statistical analysis in randomized control trials may have also caused an overestimation of the risk reduction in mortality rate of breast cancer through mammogram. ${ }^{16}$ Furthermore, even when screening mammography was associated with a reduction in breast cancer mortality rate, the screening protocol itself could only account for a third of this reduction. ${ }^{17}$ Any decreases may simply be related to increased breast health awareness, since a randomized control trial in Canada found that mammography alongside a clinical exam offered no additional reduction in mortality compared to clinical inspection alone. ${ }^{18}$

Surprisingly, mammography may actually be counterproductive. A systematic review of 19 studies comprising over two million asymptomatic women found that invasive breast cancer rates may be lower for those who do not opt for mammograms, since these unscreened women are not overdiagnosed. ${ }^{19}$ Furthermore, lesions that are highly localized in situ and are not necessarily clinically relevant are not detected in these unscreened women. Two thirds of breast cancers found by mammography are overdiagnosed, with the majority not developing into clinical disease. ${ }^{20}$ Screened women experience more stress and a greater number of biopsies, surgeries and treatments that may not offer a benefit great enough to warrant their use.

In the case of a significant history of familial breast cancer, more expensive screening techniques could be more appropriate. Magnetic resonance imaging (MRI) had a 90\% sensitivity to detect breast cancers in this high-risk population compared to $37.5 \%$ for mammography. Expensive modalities with high sensitivity such as MRI should be reserved for patients with BRCA mutations. ${ }^{21}$

\section{COLORECTAL CANCERS}

Three screening tests are available for colorectal cancer. In order of sensitivity and cost, they are: fecal occult blood testing (FOBT), sigmoidoscopy, and colonoscopy. While both sigmoidoscopy and colonoscopy can prevent most deaths from proximal colorectal cancer, only colonoscopy can help identify distal colorectal cancer. ${ }^{22}$ Colonoscopy is conventionally favoured as the primary screening tool due to its ability to detect malignant lesions throughout the colon, and to detect benign polyps that may progress to malignancy.

The Canadian Task Force on Preventive Health Care has recently revised the guidelines on colorectal cancer screening for asymptomatic low risk individuals aged 50 to 74 years. ${ }^{23}$ New guidelines recommend FOBT every 2 years and flexible sigmoidoscopy every 10 years instead of colonoscopy. Sigmoidoscopy requires significantly fewer resources and less training compared to colonoscopy. However, the task force cites a weak or limited evidence to support the benefits of colonoscopy over other modalities until further randomized clinical trials are completed. Colonoscopy currently has long wait times and is associated with higher risks, discomfort, and complications. Despite high sensitivity, colonoscopy-based screening is limited in a practical sense by lower participation rates due to patient discomfort with bowel preparation. ${ }^{24}$

One way to reduce the number of unintended adverse events due to colonoscopy is to study which populations are vulnerable to side effects and screen these individuals differently. Multiple variables were associated with unplanned hospital visits following outpatient colonoscopy due to side effects such as hemorrhage, abdominal pain and perforation. Among these variables was a history of fluid/electrolyte imbalance, psychiatric disorders and age over 65 years. These risk factors can be incorporated into a risk stratification algorithm to help fine-tune the risk-to-reward ratio of screening. ${ }^{25}$ Non-screening treatments could become alternatives or useful adjuvants to colonoscopy. Low-dose aspirin appears to have strong potential to prevent colorectal cancer even in screened patients. ${ }^{26}$ Regular-dose aspirin intake may prevent $17 \%$ of colorectal cancers in patients who have not received colonoscopy, and $8.5 \%$ in those that did. Therefore, aspirin intake could supplement less sensitive screening such as FOBT, although it has its own drawbacks and gastrointestinal side effects.

\section{FUTURE DIRECTIONS}

The underwhelming performance of routine screening tests has led to new efforts to improve guidelines and eligibility protocols. For example, to avoid costs, stress, and unnecessary treatments, many patients with a low remaining life expectancy should not be screened. A meta-analysis of survival data from the U.S., Sweden, the United Kingdom, and Denmark found that it took on average over 10 years before one death was prevented using either breast or colorectal cancer screening in 1000 individuals. ${ }^{27}$ Therefore, screening is more appropriate in individuals who are expected to live longer than 10 years after the test.

Apart from limiting screening or changing guidelines, increasing the capabilities of the screening technologies themselves may prove effective. Multitarget stool DNA testing can detect more colorectal cancers than FOBT with lower specificity. ${ }^{28}$ Improvement in detection rates of current genetic markers and the addition of new ones may make this a more viable test in the future. Urine screening may be a new modality that is effective in urologic can- 
cers. Gas chromatography alongside statistical analysis of volatile compounds was found to have high sensitivity and specificity in an early pilot study. ${ }^{29}$ Investing in the next generation of screening modalities will enhance our ability to detect malignancies more effectively, replacing conventional screening tests which have yielded modest benefits.

\section{REFERENCES}

1. Canadian Cancer Statistics Publication 2015 [internet]. Canadian Cancer Society 2013 . Available from: http://www.cancer.ca/ /media/ cancer.ca/CW/cancer\%20information/cancer\%20101/Canadian $\% 20$ cancer\%20statistics/Canadian-Cancer-Statistics-2015-EN.pdf?la=en

2. Black WC, Haggstrom DA, Welch HG. All-cause mortality in randomized trials of cancer screening. J Natl Cancer Inst. $2002 \mathrm{Feb}$ 6;94(3):167-73.

3. Saquib N, Saquib J, Ioannidis JP. Does screening for disease save lives in asymptomatic adults? Systematic review of meta-analyses and randomized trials. Int J Epidemiol. 2015 Jan; 44 (1):264-77.

4. Ankerst DP, Thompson IM. Sensitivity and specificity of prostate-specific antigen for prostate cancer detection with high rates of biopsy verification. Arch Ital Urol Androl, 2006; 78:125-9.

5. Schröder FH, Hugosson J, Roobol MJ et al. Screening and prostate cancer mortality: results of the European Randomised Study of Screening for Prostate Cancer (ERSPC) at 13 years of follow-up. Lancet. 2014 Dec;384:2027-35.

6. Moyer VA. Screening for prostate cancer: U.S. Preventive Services Task Force recommendation statement. Ann Intern Med, 2012; 157:120-34.

7. Bell N, Connor Gorber S, Shane A, et al. Recommendations on screening for prostate cancer with the prostate-specific antigen test. Cmaj, 2014;186:1225-34.

8. Banerji JS, Wolff EM, Massman JDiii et al. Prostate Needle Biopsy Outcomes in the Era of the U.S. Preventive Services Task Force Recommendation against Prostate Specific Antigen Based Screening. J Urol. 2016 Jan;195:66-73.

9. Bhuyan SS, Chandak A, Gupta N, et al. Patient-Provider Communication About Prostate Cancer Screening and Treatment: New Evidence From the Health Information National Trends Survey. Am J Mens Health. 2015 Nov 16

10. Lavallée LT, Binette A, Witiuk K, et al. Reducing the Harm of Prostate Cancer Screening: Repeated Prostate-Specific Antigen Testing. Mayo Clin Proc. 2016;91:17-22.

11. Kavanagh AM, Giles GG, Mitchell H, et al. The sensitivity, specificity, and positive predictive value of screening mammography and symptomatic status. J Med Screen. 2000;7:105-10.

12. Ohuchi N, Suzuki A, Sobue T, et al. Sensitivity and specificity of mammography and adjunctive ultrasonography to screen for breast cancer in the Japan Strategic Anti-cancer Randomized Trial (J-START): a randomised controlled trial. Lancet. 2015 Nov;387:341-8.

13. Weedon-Fekjær H, Romundstad PR, Vatten LJ. Modern mammography screening and breast cancer mortality: population study. BMJ. 2014;348.

14. Autier P, Boniol M, Gavin A, et al. Breast cancer mortality in neighbouring European countries with different levels of screening but similar access to treatment: trend analysis of WHO mortality database. BMJ.2011;343.

15. Mukhtar TK, Yeates, DR, Goldacre MJ. Breast cancer mortality trends in England and the assessment of the effectiveness of mammography screening: population-based study. J R Soc Med. 2013;106:234-42.

16. Autier P, Boniol M, Smans M, et al. Statistical analyses in Swedish randomised trials on mammography screening and in other randomised trials on cancer screening: a systematic review. J R Soc Med. 2015;108;440-50.

17. Kalager M, Zelen M, Langmark F et al. Effect of screening mammography on breast-cancer mortality in Norway. N Engl J Med. 2010;363:1203-10.

18. Taghipour S, Banjevic D, Fernandes J, et al. Incidence of invasive breast cancer in the presence of competing mortality: the Canadian National Breast Screening Study. Breast Cancer Res Treat. 2012;134:839-51.

19. Cutler W, Burki R, Kolter J, et al. Invasive Breast Cancer Incidence in 2,305,427 Screened Asymptomatic Women: Estimated Long Term Outcomes during Menopause Using a Systematic Review. PLoS One. 2015;10: e0128895.

20. Zahl PH, Maehlen J. [Overdiagnosis in mammography screening] Tidsskr Nor Laegeforen. 2004 Sep 9;124(17):2238-9.

21. Riedl CC, Luft N, Bernhart C, et al. Triple-Modality Screening Trial for Familial Breast Cancer Underlines the Importance of Magnetic Resonance Imaging and Questions the Role of Mammography and Ultrasound Regardless of Patient Mutation Status, Age, and Breast Density. J Clin Oncol. 2015;33:1128-35.

22. Brenner H, Stock C, Hoffmeister M. Effect of screening sigmoidoscopy and screening colonoscopy on colorectal cancer incidence and mortality: systematic review and meta-analysis of randomised controlled trials and observational studies. BMJ. 2014;348.

23. Care, C.T.F.o.P.H. Recommendations on screening for colorectal cancer in primary care. CMAJ. 2016;188:340-8.

24. Kuipers EJ, Spaander MCW. Colorectal Cancer Screening by Colonoscopy, CT-Colonography, or Fecal Immunochemical Test. J Natl Cancer Inst. 2016;108

25. Ranasinghe I, Parzynski, CS, Searfoss R, et al. Differences in Colonoscopy Quality Among Facilities: Development of a Post-Colonoscopy Risk-Standardized Rate of Unplanned Hospital Visits. Gastroenterology. 2016;150:103-13.

26. Cao Y, Nishihara R, Wu, K, et al. Population-wide impact of longterm use of aspirin and the risk for cancer. JAMA Oncology. 2016 Jun 1;2(6):762-9.

27. Lee SJ, Boscardin WJ, Stijacic-Cenzer I, et al. Time lag to benefit after screening for breast and colorectal cancer: meta-analysis of survival data from the United States, Sweden, United Kingdom, and Denmark. BMJ. 2013;346.

28. Berger BM, Schroy Iii PC, Dinh TA. Screening for Colorectal Cancer Using a Multitarget Stool DNA Test: Modeling the Effect of the Intertest Interval on Clinical Effectiveness. Clinical Colorectal Cancer. 2015 Dec 18; pii: S1533-0028(15)00154-1.

29. Raphael BMA, Ben de Lacy C, Paul W, et al. The use of a gas chromatography-sensor system combined with advanced statistical methods, towards the diagnosis of urological malignancies. Journal of Breath Research. 2016 Feb 11; 10(1): 017106. 